\title{
Correlation between Vertebral Left Atrial Size and NT-proBNP in Dogs with Myxomatous Mitral Valve Disease
}

\section{Dong-Jin Chae \\ Sung-Hyun Han \\ Kunho Song*}

College of Veterinary Medicine, Chungnam National University, Daejeon 34134, South Korea
*Correspondence: songkh@cnu.ac.kr

\section{ORCID}

Dong-Jin Chae:

https://orcid.org/0000-0002-0720-1898

Sung-Hyun Han:

https://orcid.org/0000-0003-2512-1442

Kunho Song:

https://orcid.org/0000-0001-8478-2035

Copyright $\odot$ The Korean Society of Veterinary Clinics
Abstract The purpose of this study was to investigate the correlation between vertebral left atrial size (VLAS) and NT-proBNP levels. Sixty-three dogs with myxomatous mitral valve disease (MMVD) were recruited for this study. The MMVD group was classified according to the ACVIM stage based on medical history, clinical signs, and thoracic radiography and echocardiography findings. The N-terminal pro-B-type natriuretic peptide (NT-proBNP) levels were measured using fluorescent immunoassay. VLAS and VHS values were measured using a digital caliper in the right lateral view. There were significant differences in VLAS for each American college of veterinary internal medicine (ACVIM) stage $(p<0.01)$. As the ACVIM stage increased, it tended to increase. Vertebral heart score (VHS) also showed a tendency to increase with the ACVIM stage $(p<0.01)$. VLAS was divided into three groups: VLAS $<2.6,2.6 \leq$ VLAS $<3.1$, and VLAS $\geq 3.1$. NT-proBNP values were significantly different between the three groups $(p<0.01)$, and VLAS and NT-proBNP values showed a strong positive correlation ( $r=0.756, p<0.01)$. In conclusion, there were significant differences among ACVIM stages B1 and B2, $B 1$ and C-D, and B2 and C-D groups regarding VLAS. Furthermore, NT-proBNP increased proportionally as VLAS increased. Measurements of VLAS may be helpful in the diagnosis of MMVD in addition to the existing radiological and echocardiographic methods.

Key words NT-proBNP, VLAS, MMVD, dog. 


\section{Introduction}

Myxomatous mitral valve disease (MMVD) is the most common cardiac disease in dogs. Clinical evidence of degenerative valvular disease is detected in approximately $30 \%$ of dogs aged $\geq 13$ years (5). MMVD is a progressive disease, and subtle changes in the valve structure precede the development of clinically evident valvular dysfunction. MMVD is extremely common, but progresses at a rate that varies greatly among individuals (1). When the mitral valve is incompetent, mitral valve regurgitation (MR) can occur, and development of clinically consequential MR may lead to enlargement of the cardiac silhouette. In the overwhelming majority of cases, left atrial enlargement precedes the development of congestive heart failure (CHF). A diagnosis of left-sided CHF secondary to MMVD can rarely be supported in the absence of radiographic left atrial enlargement (1). Although echocardiography is a useful tool to detect hypertrophy or hematological abnormalities in the left atrium and left ventricle of MMVD patients, it requires accurate measurement by a skilled expert and is time-consuming and expensive.

In most cases, thoracic radiography provides the most useful diagnostic and prognostic information in patients with MMVD. Thoracic radiography not only assesses cardiac size but also allows visualization of the pulmonary vessels and parenchyma.

The American college of veterinary internal medicine (ACVIM) stage $(p<0.01)$. As the American college of veterinary internal medicine (ACVIM) guidelines, updated in 2019, present criteria for determining MMVD Stage B. Dogs in Stage B have a structural abnormality of the valve, but have never had clinical signs of heart failure associated with this disease. Stage B can be further divided into stage B1 or B2 according to several criteria (6).

Based on the results of a clinical trial, ACVIM Stage B2 indicates asymptomatic MMVD causing mitral regurgitation (MR) severe enough to result in cardiac remodeling (LA and LV enlargement) sufficient to recommend treatment before the onset of clinical signs (14). Dogs in this category should meet the following criteria: murmur intensity $\geq 3 / 6$, echocardiographic left atrial-to-aortic ratio $(\mathrm{LA} / \mathrm{Ao}) \geq 1.6$ (15), normalized left ventricular internal diameter in diastole (LVIDDN) $\geq 1.7$ (2), and thoracic radiographic vertebral heart score (VHS) $>10.5$ (8). If echocardiography is unavailable, VHS $\geq 11.5$, or evidence of a rising interval change revealed by thoracic radiography can replace echocardiography to confirm Stage B2 (11).

Vertebral left atrial size (VLAS), a new index of radiographic left atrial enlargement, provides a quantitative method for estimating left atrial size $(1,10,11)$. Measured on either the right or left lateral radiograph by drawing a line from the center of the most ventral aspect of the carina to the most caudal aspect of the LA, where it intersects with the dorsal border of the caudal vena cava, which is then transposed to the cranial edge of the 4th thoracic vertebral body (11). Studies are ongoing to determine a VLAS value that accurately predicts B2 remodeling, but in the absence of echocardiography, VLAS $\geq 3$ likely indicates Stage B2 MMVD (11).

B-type natriuretic peptide (BNP), produced by myocardial tissue in response to increased pressure and wall stress, is a marker of cardiac dysfunction and heart failure $(9,14)$. Mature BNP has a short half-life (20 min), and clinical assays that target prohormones (NT-proBNP) are more useful because they have longer half-lives (120 min) (3). The following guidelines appear useful for canine NT-proBNP: NT-proBNP >800 pmol/ $L$ has a high probability of heart disease, but not necessarily congestive heart failure, NT-proBNP $>1800 \mathrm{pmol} / \mathrm{L}$ can be diagnosed as heart disease, and NT-proBNP $>2700 \mathrm{pmol} / \mathrm{L}$ is consistent with CHF (4).

Therefore, the purpose of this study was to identify the correlation between VLAS and NT-proBNP levels in dogs with MMVD.

\section{Materials and Methods}

\section{Animals}

This study included 63 dogs with MMVD. All dogs were client-owned dogs and participated in the local animal clinic between February 2017 and June 2021. Before initiating the experiment, informed consent was obtained from the participants' owners. MMVD was diagnosed based on findings from physical examination, thoracic radiography, and echocardiography. All patients were classified as MMVD stage B1 $(n=18), B 2(n=18)$, or C-D $(n=27)$ according to the ACVIM guidelines. NT-proBNP values were investigated in all dogs in relation to VLAS.

\section{Blood sample collection}

Blood samples of $2 \mathrm{~mL}$ of whole blood was collected from the jugular vein or cephalic vein of the fasting dogs. Following collection, samples were left to coagulate for $10 \mathrm{~min}$, then centrifuged at 1,500 $\times \mathrm{g}$ for $10 \mathrm{~min}$, and the centrifuged serum was separated into Eppendorf tubes.

\section{NT-proBNP assays}

NT-proBNP values were measured using a fluorescent immunoassay system (BIONOTE, V200, S. Korea). The BIONOTE test results showed a high correlation with the IDEXX's CAR- 
DIOPET (ELISA) test result (R-squared value $>0.90, \mathrm{~N}=20$, evaluated by URIKA LLC of Mukilteo, WA). The NT-proBNP reference of this machine ranged from 500 to $10,000 \mathrm{pmol} / \mathrm{L}$. These systems suggest that the reference range NT-proBNP $<900 \mathrm{pmol} / \mathrm{L}$ is normal, $900-1800 \mathrm{pmol} / \mathrm{L}$ suspected heart disease, and $>1800 \mathrm{pmol} / \mathrm{L}$ highly suspected heart disease.

\section{Thoracic radiography}

Thoracic radiography was used to obtain ventrodorsal, right lateral, and left lateral views in all patients. VLAS and VHS values were measured for each dog. Both VLAS and VHS were measured using a digital caliper in the right lateral view. The method of measuring VLAS was as follows: drawing a line from the center of the most ventral aspect of the carina to the most caudal aspect of the left atrium where it intersects with the dorsal border of the caudal vena cava, that line then is transposed to the cranial edge of the 4th thoracic vertebral body.

\section{Echocardiography}

Echocardiography was performed using a LOGIQ P9 (Phillips, Bothwell WA, 98041 USA) to detect mitral valve degeneration and mitral regurgitation. Mitral valve prolapses were identified by color Doppler imaging in the left apical 4-chamber view and two-dimensional echocardiography. The left ventricular end diastolic diameter in diastole (LVIDD) was obtained in M-mode. Left ventricular end-diastolic diameter normalized for body weight (LVIDDN) was evaluated using the formula (LVIDDN = LVIDD $[\mathrm{cm}] /$ weight $\left.[\mathrm{kg}]^{0.294}\right)$. The LA/ Ao ratio was also measured in the right parasternal two-dimensional short-axis view.

\section{Statistical analysis}

Statistical analysis was performed using a commercially available computer-based software program (IBM SPSS

Table 1. Demographic distribution and radiographic, echocardiographic data values according to ACVIM stage

\begin{tabular}{lllc}
\hline \multicolumn{1}{c}{ Group } & \multicolumn{1}{c}{$\begin{array}{c}\text { Stage B1 } \\
(\mathbf{n}=\mathbf{1 8})\end{array}$} & \multicolumn{1}{c}{$\begin{array}{c}\text { Stage } \mathbf{B 2} \\
(\mathbf{n}=\mathbf{1 8})\end{array}$} & $\begin{array}{c}\text { Stage C-D } \\
(\mathbf{n}=\mathbf{2 7})\end{array}$ \\
\hline Age $(\mathrm{yr})$ & $7.41(1-16)$ & $11.4(4.5-17.3)$ & $12.2(4-17.4)$ \\
Weight $(\mathrm{kg})$ & $6.81(1.86-33)$ & $7.28(2.66-15.4)$ & $3.81(2.12-14.2)$ \\
VHS & $9.78(9-10.5)$ & $10.9(10.3-13)$ & $11.57(10.5-14)$ \\
VLAS & $2.09(1.7-2.5)$ & $2.54(2.2-3)$ & $3.1(2.6-4)$ \\
LA/Ao ratio & $1.25(1.11-1.48)$ & $1.63(1.12-2.12)$ & $2.18(1.56-3.2)$ \\
\hline
\end{tabular}

ACVIM, American College of Veterinary Internal Medicine; VHS, Vertebral heart size; VLAS, Vertebral left atrial size; LA/Ao ratio, left atrial-to-aortic ratio.
Statistics 24.0.0, SPSS Inc., USA). The values of each group, including age, weight, VHS, VLAS, and LA/Ao ratio, were reported as medians and interquartile ranges (IQR). The values among the three groups were compared using ANOVA, and post-hoc tests were conducted using the Bonferroni correction. The correlation between VLAS and NT-proBNP was analyzed using ANOVA, and post-hoc tests were conducted using the Bonferroni correction. Additionally, Pearson correlation coefficients $(r)$ were used to confirm the correlation between VLAS and NT-proBNP levels. A $p$ value of $<0.05$ was considered statistically significant.

\section{Results}

A total of 63 dogs were used in the study and were classified according to the two criteria. The first classification was according to the ACVIM stage criteria, and the second was based on age, weight, VHS, VLAS, and LA/Ao ratio. The details for each classification are summarized in Table 1. The stage B1 group consisted of 18 dogs, and the Stage B2 and C-D groups comprised 18 dogs and 27 dogs, respectively. The radiographic and echocardiographic values of each ACVIM stage are summarized in Table 1.

When comparing VLAS, VHS, and NT-proBNP between each group, VLAS values were significantly different between $B 1$ and B2 groups $(p<0.01)$, B2 and C-D groups $(p<0.01)$, and $B 1$ and C-D groups $(p<0.01)$ (Fig. 1). VHS values were significantly different between B1 and B2 groups ( $p<0.01)$, $B 2$ group and C-D group $(p<0.05)$, and B1 and C-D groups ( $p$ $<0.01$ ) (Fig. 2). As for NT-proBNP among each group, only the B2 group and the C-D group showed a significant difference $(p<0.01)$ (Fig. 3).

The correlation between VLAS and NT-proBNP levels is

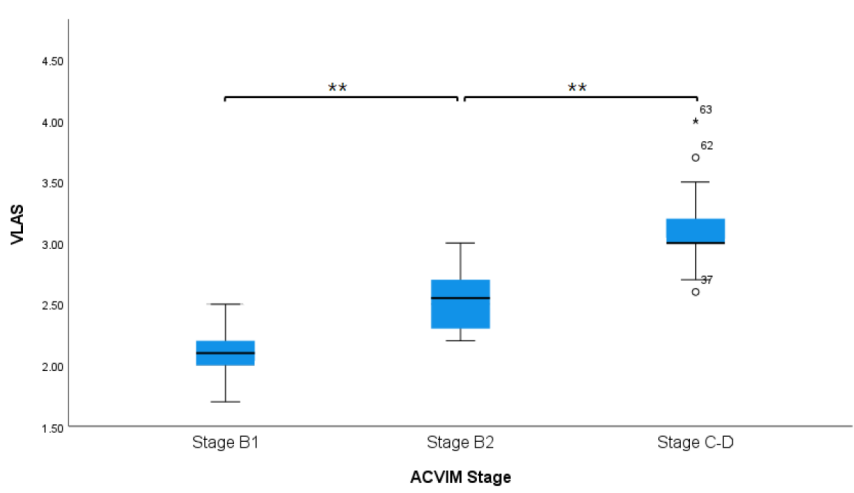

Fig. 1. Evaluation of the correlation VLAS according to the ACVIM stage. VLAS, Vertebral left atrial size; ACVIM, American College of Veterinary Internal Medicine. VLAS showed a significant difference. ${ }^{* *} p<$ 0.01 . 


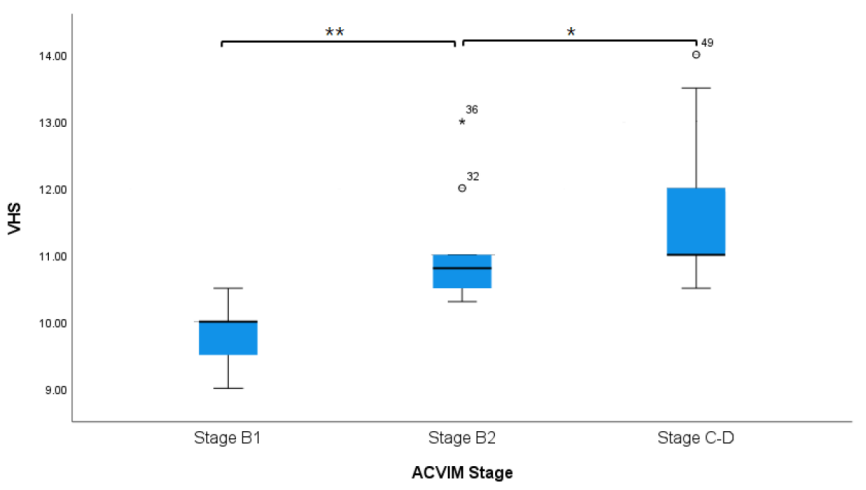

Fig. 2. Evaluation of the correlation VHS according to ACVIM stage. VHS, Vertebral heart size; ACVIM, American College of Veterinary Internal Medicine. VHS showed a significant difference. ${ }^{*} p<0.05,{ }^{* *} p$ $<0.01$.

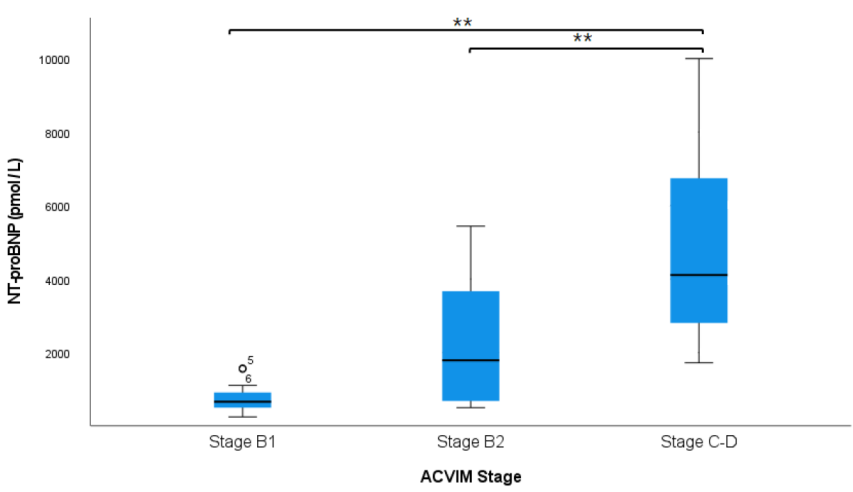

Fig. 3. Evaluation of the correlation NT-proBNP according to ACVIM stage. NT-proBNP, N-terminal pro B-type natriuretic peptide; ACVIM, American College of Veterinary Internal Medicine. ${ }^{* *} p<0.01$

summarized in Fig. 4. Sixty-three dogs were classified into three groups: VLAS $<2.6,2.6 \leq \mathrm{VLAS}<3.1$, and VLAS $\geq 3.1$ group. The NT-proBNP values were analyzed for each group. There was a significant difference between the VLAS $<2.6$ and $2.6 \leq$ VLAS $<3.1$ groups $(p<0.01)$. Also, there was a significant difference between the $2.6 \leq$ VLAS $<3.1$ group and the VLAS $\geq 3.1$ group ( $<<0.01$ ). We found a strong positive correlation between VLAS and NT-proBNP levels (Fig. 5). The correlation coefficient between VLAS and NT-proBNP was 0.756 , and the R-squared value was 0.571 , which is approximately $57 \%$ of the explanatory power.

\section{Discussion}

In this study, patients with MMVD were divided into B1, $B 2$, and C-D groups according to the ACVIM criteria, and their age, weight, VHS, VLAS, LA/Ao ratio, and NT-proBNP were compared. In a recent study by Kim et al. (7), VLAS

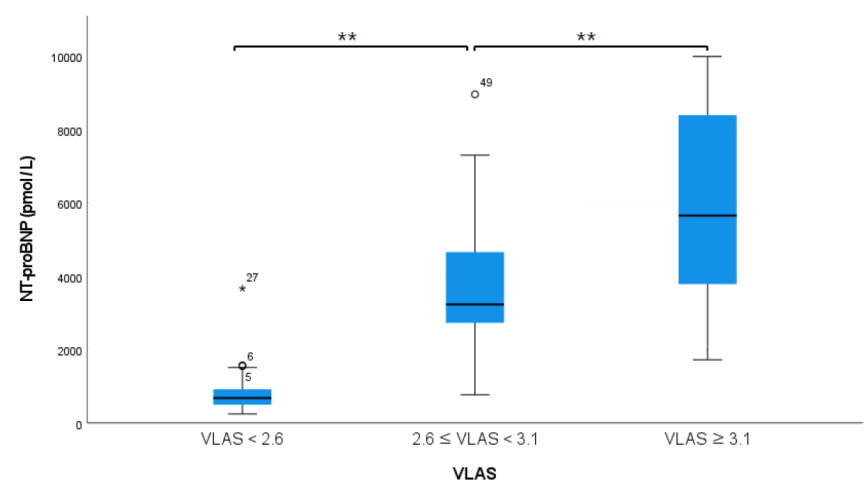

Fig. 4. Evaluation of the correlation between VLAS and NT-proBNP in three groups. VLAS, Vertebral left atrial size; NT-proBNP, N-terminal pro B-type natriuretic peptide. ${ }^{* *} p<0.01$.

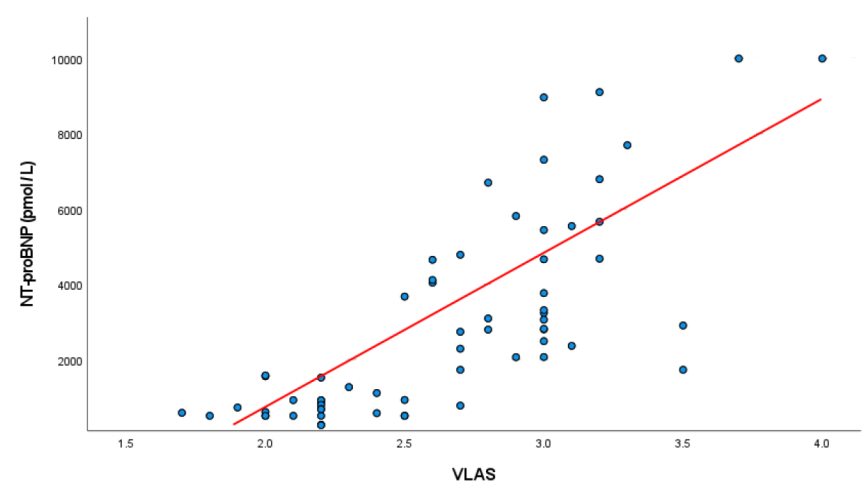

Fig. 5. VLAS and NT-proBNP have a strong positive correlation, and the R-squared value is 0.571 . VLAS, Vertebral left atrial size; NT-proBNP, $\mathrm{N}$-terminal pro B-type natriuretic peptide $(p<0.01)$.

showed a tendency to increase as the ACVIM stage increased, and the same results were found in this study. VHS also showed a tendency to increase with increasing ACVIM stage, which is consistent with the results of previous studies $(6,8)$. This result suggests that VLAS can be used for the ACVIM stage criteria in addition to the currently used VHS. In a recent study by Park et al. (13), NT-proBNP showed a tendency to increase as the ACVIM stage increased, and similar results were found in this study. In addition, when the values of VLAS were divided into three groups (VLAS $<2.6,2.6 \leq$ VLAS <3.1, and VLAS $\geq 3.1$ ), NT-proBNP was also found to have a significant difference for each group. There was a strong positive correlation between VLAS and NT-proBNP levels.

In a recent study, the median VLAS in a healthy dog was 1.9, and the reference interval was 1.4-2.2. In addition, this value is not affected by sex, age, or weight (16). In addition, studies are being conducted to determine the cut-off value of VLAS corresponding to ACVIM stage B2, which is the recommend- 
ed stage to start medical management. In a recent study by Mikawa et al. (11), VLAS $\geq 2.5$, exhibited the highest sensitivity (sensitivity, 100\%; specificity, 78\%), and a VLAS $\geq 3.1$, exhibited the highest specificity (sensitivity, 47\%; specificity, $100 \%$ ). They reported that a VLAS cutoff value of 2.6, provided the greatest diagnostic accuracy for the identification of dogs with ACVIM stage B2 MMVD (area under the curve, 0.96; sensitivity, 95\%; specificity, 84\%). In addition, a VLAS cutoff value of 2.5 , could be used to identify dogs that may benefit from echocardiography to determine if they have reached ACVIM stage B2.

In this study, VLAS was significantly different for each ACVIM stage, and in stage B2, it had an average value of 2.54, supporting the above results. NT-proBNP is secreted when the myocardial tissue is compressed and under stress. It is used to diagnose heart disease or to evaluate the prognosis of heart failure. In a recent study by Ogawa et al. (12), the cut-off value of NT-proBNP, which determines ACVIM stage B2 or higher, was $1453.00 \mathrm{pmol} / \mathrm{L}$ (area under the curve, 0.75 ; sensitivity, $80 \%$; specificity, $64.7 \%$ ). However, there was no significant difference between the B1 and B2 and $B 2$ and C-D groups. In addition, the correlation coefficient of NT-proBNP with VLAS was 0.534 . In this study, the median NT-proBNP level of the ACVIM stage B2 group was 2,230 pmol/L. However, only the B2 and C-D groups showed significant differences. However, when the groups were divided based on VLAS, NT-proBNP showed a significant difference between each group. This means that VLAS and NT-proBNP can be helpful in the diagnosis of MMVD and evaluation of prognosis.

This study had two limitations. First, the effects of the administered drugs, such as pimobendan, diuretics, and angiotensin-converting enzyme inhibitor were not considered. These drugs are involved in myocardial contractility, preload, and afterload, which can affect myocardial stress and heart size. Second, there was no distinction between diseases other than MMVD that could affect the outcomes of VLAS, VHS, and NT-proBNP. Many other diseases can affect heart size and NT-proBNP levels. However, these factors were not sufficiently explored in this study. In future, a longitudinal study of VLAS and NT-proBNP in dogs with MMVD is needed.

In conclusion, there were significant differences among ACVIM stages B1 and B2, B1 and C-D, and B2 and C-D groups regarding VLAS. Furthermore, NT-proBNP increased proportionally as VLAS increased. Therefore, in evaluating the size of the heart, it would be beneficial to measure VLAS and NT-proBNP in addition to the existing radiological and echocardiographic methods, thereby employing it as an ad- ditional diagnostic tool.

\section{Conflicts of Interest}

The authors have no conflicting interests.

\section{References}

1. Abbott JA. Acquired valvular disease. In: Tilley L, Smith F, Oyama M, Sleeper M, editors. Manual of canine and feline cardiology. 4th ed. St. Louis: Elsevier Sounders. 2008: 110-138.

2. Cornell CC, Kittleson MD, Della Torre P, Häggström J, Lombard $\mathrm{CW}$, Pedersen HD, et al. Allometric scaling of M-mode cardiac measurements in normal adult dogs. J Vet Intern Med 2004; 18 311-321.

3. Cote E, MacDonald KA, Meurs KM, Sleeper MM. Feline cardiology. Arnes: Wiley-Blackwell. 2011: 69-75.

4. de Lima GV, Ferreira FDS. N-terminal-pro brain natriuretic peptides in dogs and cats: a technical and clinical review. Vet World 2017; 10: 1072-1082.

5. Ishikawa T, Tanaka R, Suzuki S, Saida Y, Soda A, Fukushima R, et al. Daily rhythms of left atrial pressure in beagle dogs with mitral valve regurgitation. J Vet Intern Med 2009; 23: 824-831.

6. Keene BW, Atkins CE, Bonagura JD, Fox PR, Häggström J, Fuentes $V L$, et al. ACVIM consensus guidelines for the diagnosis and treatment of myxomatous mitral valve disease in dogs. J Vet Intern Med 2019; 33: 1127-1140.

7. Kim SH, Seo KW, Song KH. An assessment of vertebral left atrial size in relation to the progress of myxomatous mitral valve disease in dogs. J Vet Clin 2020; 37: 9-14.

8. Lamb CR, Wikeley H, Boswood A, Pfeiffer DU. Use of breed-specific ranges for the vertebral heart scale as an aid to the radiographic diagnosis of cardiac disease in dogs. Vet Rec 2001; 148 : 707-711.

9. Latini R, Masson S. NT-proBNP: a guide to improve the management of patients with heart failure. EJIFCC 2013; 24: 78-84.

10. Malcolm EL, Visser LC, Phillips KL, Johnson LR. Diagnostic value of vertebral left atrial size as determined from thoracic radiographs for assessment of left atrial size in dogs with myxomatous mitral valve disease. J Am Vet Med Assoc 2018; 253: 1038-1045.

11. Mikawa S, Nagakawa M, Ogi H, Akabane R, Koyama Y, Sakatani $A$, et al. Use of vertebral left atrial size for staging of dogs with myxomatous valve disease. J Vet Cardiol 2020; 30: 92-99.

12. Ogawa M, Hori Y, Kanno N, Iwasa N, Toyofuku T, Isayama N, et al. Comparison of $\mathrm{N}$-terminal pro-atrial natriuretic peptide and three cardiac biomarkers for discriminatory ability of clinical stage in dogs with myxomatous mitral valve disease. J Vet Med Sci 2021; 83: 705-715.

13. Park JS, Park JH, Seo KW, Song KH. Correlation between NT-proB- 
NP and lipase levels according to the severity of chronic mitral valve disease in dogs. J Vet Sci 2019; 20: e43.

14. Silverstein DC. Small animal critical care medicine. 2nd ed. St. Louis: Elsevier Sounders. 2015: 213-217.

15. Strohm LE, Visser LC, Chapel EH, Drost WT, Bonagura JD. Two-dimensional, long-axis echocardiographic ratios for assessment of left atrial and ventricular size in dogs. J Vet Cardiol 2018; 20: 330 342.

16. Vezzosi T, Puccinelli C, Tognetti R, Pelligra T, Citi S. Radiographic vertebral left atrial size: a reference interval study in healthy adult dogs. Vet Radiol Ultrasound 2020; 61: 507-511. 